\title{
Advanced Elemental Analysis with ED-EPMA, WD-EPMA and $\mu$-XRF at a SEM
}

\author{
V.-D. Hodoroaba, ${ }^{*}$ T. Salge, ${ }^{* *}$ R. Terborg** and V. Rackwitz* \\ * BAM Federal Institute for Materials Research and Testing, 12200 Berlin, Germany \\ ** Bruker Nano GmbH, 12489 Berlin, Germany
}

It is a latent wish of any SEM/EDS (scanning electron microscope with an energy dispersive spectrometer) analyst to "see more" of the analyzed specimen, i.e. to improve the existing analytical figures of merit.

One key issue are the relatively poor limits of detection (not below 0.1 mass-\%) provided by energy dispersive X-ray spectrometry (EDX) with the conventional electron excitation (ED-EPMA). This is a consequence of relatively low peak-to-background ratios and reduced energy resolution when compared to wavelength dispersive spectrometry (WD-EPMA). Recent technological developments make possible to equip the SEM with a wavelength dispersive spectrometer (WDS), so that significantly better energy resolution can be attained [1,2]. Also a relative new product that can be easily attached to a SEM/EDS system is a micro-focus X-ray source [3]. Hence, it is possible to perform (micro-focus) X-ray fluorescence spectrometry ( $\mu$-XRF) and take advantage of the enhanced peak-to-background ratios (well suited for trace analysis). However, there are also some disadvantages: an increased measurement time and excitation with a high current in the 10s of nA range are usually required for WDS. $\mu$-XRF provides more bulk information and poor limits of detection for light elements. By combining the advantages of these analytical techniques "seeing more" becomes possible.

Another relevant analytical aspect is to lower the spatial resolution for elemental analysis. This makes it necessary to work with low excitation voltages at the SEM and evaluate the low energy Xray lines. In order to separate overlapping peaks, the line deconvolution algorithms incorporated in the quantification ED-EPMA software are of crucial importance. In addition, elements which are part of small, differently composed inclusions can be identified in two ways: (1) the fast $\mu$-XRF bulk analysis technique is sensitive to trace elements; (2) the maximum pixel spectrum function [4] synthesizes a spectrum out of a hyperspectral EDS database which consists of the highest count level found in each spectrum channel.

Examples of the topics described above will be discussed using representative application examples including analysis of a sintered hard ceramic material composed of three phases $\mathrm{TiB}_{2}, \mathrm{TiC}$, and $\mathrm{SiC}$, see Fig. 1 and Fig. 2. Special attention will be paid to the accurate separation of W-L from Si-K or of Ti-L from N-K with ED- and WD-EPMA.

\section{References}

[1] D. Redfern, A. Sandborg, Microsc. Microanal. 11 (Suppl 2) (2005) 468.

[2] C. van Hoek, M. Koolwijk, Microchim. Acta 161 (2008) 161.

[3] M. Procop, V.-D. Hodoroaba, Microchim. Acta 161 (2008) 413.

[4] D.S. Bright, D.E. Newbury, J. Microsc. 216 (2004) 186.

[5] Dr. R. Wäsche (BAM) is gratefully acknowledged for producing the ceramic sample. 


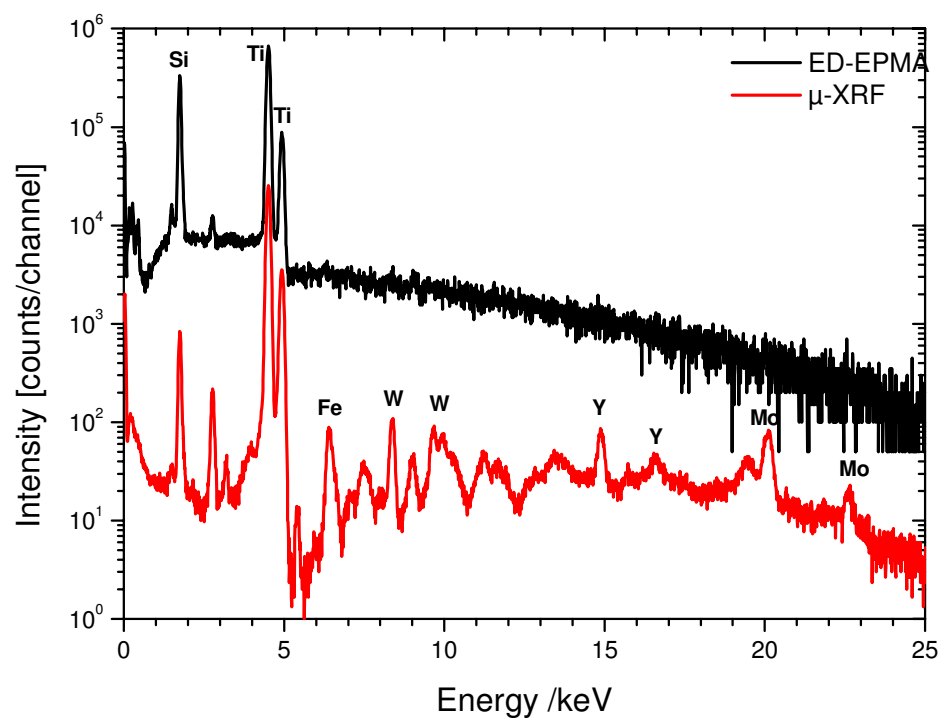

FIG. 1. ED-EPMA spectrum (in black) and $\mu$-XRF spectrum (in red) of a sintered hard ceramic material composed of three phases $\mathrm{TiB}_{2}$, TiC, and $\mathrm{SiC}$. The XRF spectrum indicates traces of $\mathrm{Fe}, \mathrm{W}$ and $\mathrm{Y}$.
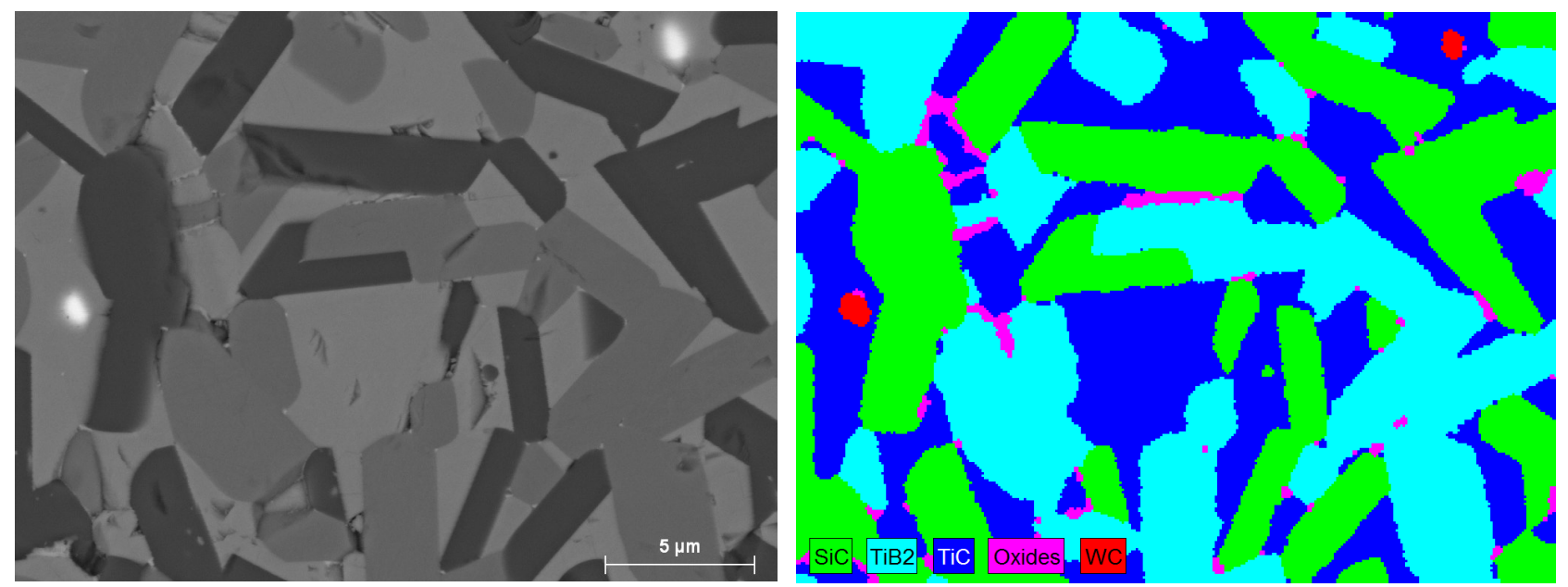

FIG. 2. Left: BSE image of the analyzed area showing three major phases (dark, medium and light grey) and a minor, bright phase below $1 \mu \mathrm{m}$ in size; Right: ED-EPMA phase map showing the distribution of major and minor components. 